\title{
SCHOOL STRESS COPING STRATEGIES CHECKLIST
}

This paper deals with school stress and describes coping strategies used in load situations. Empirical research reveals what extent of stress pupils of elementary schools perceive as well as what coping strategies are most commonly utilised. Based on the findings we can conclude that the extent of the stress perceived by the sample group of school children is moderate and they resort to coping strategies such as "avoidant action" and "distracting action".

Key words: stress, stress in schools, coping strategies, CCSC questionnaire

\section{Introduction}

The word stress has become more frequent notion not only among experts but also in general public. We deal with stress on a daily basis at school, at work, even at active recreation, hence everywhere where performance, success and "perfection" are of crucial importance. Stress has become a natural part of life of adult individuals, however recently it has been affecting younger generations to a growing extent. A number of theoretical and practical findings enable us to assume that the most contributing factor generating stress in children is school. A profound research of "school stress" has been conducted in the last thirty years both in our country as well as abroad (Ayers, T.A., Sadler, I.N. 1996, Kyriacov 2001, Mares 2000, Koubekova 2000, Bratska 1992, 2004, Hanzlova, M. - Macek, P. 2008, Fickova, E. 2001, Sarmany Schuller 1999, etc.)

School stress can be defined as a negative emotional experience, e.g. anxiety, anger, depression, frustration, resulting from certain aspects of learning. The term school stress refers to the type of stress induced at school, and hence distinction must be made from other sources of stress, e.g. fear for parents who continuously argue or a long-term impaired health condition etc. Nevertheless the stress that arises outside school that has a significant impact on a pupil's temper and behaviour at school but it must not be considered the cause of school stress. [14] School stress mostly results from concerns and worries due to school and demands laid by the school.

Manifested symptoms of stress are not only mental (neurotical symptoms, anxiety, tension, fear, aggressiveness), but also physical (enuresis, headache, stomachache, allergies, and various other indef- inite symptoms). Immune system is also significantly affected by stress, which may lead to deteriorating health condition. [11]

School places high demands on the personality of a pupil, which then plays an important role in coping with stress. Coping with stress are exceptionally important skills for pupils. Discovering coping strategies can contribute to reducing stress in schools. Determining appropriate requirements and respecting abilities and interests of a child can help reduce and eliminate pressure.

Definition of the term "coping" is not unified in technical literature. The term "coping" has its origin in Greek "colaphus" (box on the ear). General meaning of the word refers to an effort to solve problems, handle conflicts or arguments. The English word coping implies an ability to deal with a difficult, almost unmanageable situation. [13]

Most frequently cited definition by R. S. Lazarus and S. Folkmanova (1984) describes coping as an ever-changing cognitive and behavioural effort of an individual to handle, tolerate or reduce demands that burden or even exceed their mental abilities. [12]

Several authors pointed out the differences between defence mechanisms and coping strategies. [12, p. 537] According to Erickson (1997), common features of defence and coping reactions are reducing stress and controlling emotions, dynamic nature; they are both potentially reversible and developable throughout people's lives and particular elements can be distinguished in them. However, they differ in certain aspects; defence reactions include implicit operations in contrast to coping reactions, they are activated intrapsychically, an individual is not aware of them; they are determined by personality features and are based on instinctive behav-

\footnotetext{
* Katarina Mackova ${ }^{1}$, Andrea Gavlakova ${ }^{2}$

${ }^{1}$ Department of Paedagogical Studies, Faculty Science, University of Zilina, Slovakia, E-mail: katarina.mackova@fpv.uniza.sk

${ }^{2}$ Department of Foreign Languages, Faculty Science, University of Zilina, Slovakia
} 
iour. They are not preceded by evaluation of the situation, they lead to automatic behaviour and are more difficult to observe. Coping is considered to be a personality feature or a behavioural style. [3]

\section{Brief Overview of Copin Strategies Research}

Much research has been carried out with the aim to discover most often used coping strategies. Amirkhan (1990) based his work on the presumption that despite a great number of existing and well-defined coping strategies their actual value does not correspond to the theoretical grounds and assumptions. Amirkhan made an attempt to bridge deductive approach (prefabricated taxonomies of coping behaviour categories) and inductive approach (elaborating response clusters constituting general categories based on the coping behaviour documentation) by studying conjunction between them. [16]

CSI scale (Coping Strategy Indicator, J. H. Amirkhan, 1990) was created on basis of a factor analysis in order to establish three factors corresponding to three fundamental methods. They are used as a primary source to cope with stressful experiences. The three strategies are listed below:

- Instrumental strategy, relates to problem directed approach

- Seeking social support relates to the basic human need for human contact through a process of actively turning to others for comfort, help and advice,

- Avoidance strategy, relates to both physical and psychological escape. [16] KIDCOPE scale (Spirito, 1988) identifies ten coping strategies:

Distraction, social withdrawal, cognitive restructuring, self-criticism, blaming others, problem solving, emotional regulation (positive and negative), wishful thinking, social support, and resignation.

Fickova, Ruiselova (2001) carried out research based on KIDCOPE scale. A sample of 1 st and 3 rd year grammar school students in Bratislava (aged 14 - 17) were used in their analysis to identify coping strategies in stressful situations.

The questionnaire verified presumed differences between boys and girls. Girls preferred problem solving, wishful thinking, social support, cognitive restructuring and boys gave preference to problem solving, cognitive restructuring, wishful thinking and self-criticism. Both male and female adolescents prefer problem solving but differ in seeking social support which rates ninth in boys. Blaming others and negative emotional regulation are the least frequently utilised strategies by both girls and boys. [9]

COPE, developed by C.S. Carver, M.F: Scheier and J. F. Weintraub (1989), is another type of a questionnaire comprising 60 items, 4 items for 15 coping strategies. It is based on the Lazarus's stress and coping model (Lazarus, 1966), self-regulation model (Carver, Scheier, 1985) and results of research presenting various levels of coping. The questionnaire is comprised of the following strategies:

Active coping, planning, suppression of competing activities, restraint coping, seeking social support for instrumental reasons, seeking social support for emotional reasons, positive interpretation and growth, acceptance, turning to religion, focus on venting of emotions, denial, behavioral disengagement and mental disengagement.

E. Fickova (1992) described and elaborated Slovak version of COPE questionnaire. A sample of $2421^{\text {st }}$ and $3^{\text {rd }}$ year grammar school students were studied in the analysis. The results showed that girls mostly recurred to seeking social support for emotional reasons, positive interpretation and growth, seeking social support for instrumental reasons and acceptance. Boys preferred positive interpretation and growth, planning, active coping and acceptance. Both sexes congruently tended not to resort to alcohol and drug abuse, behavioural disengagement, turning to religion and denial. The most significant and statistically considerable divergence regards social support for emotional reasons utilised by girls in the first place and by boys in the tenth place. [9]

CISS (Coping Inventory for Stressful Situations), elaborated by N. S. Endler and J. D. A. Parker (1990), is another coping strategies questionnaire comprised of 48 task oriented (efforts to change and solve problem situation), emotion oriented (efforts to reduce stress) and avoidance coping items. The analysis was executed by E. Fickova (1992). The results revealed only slightly higher coping indicators' values in girls. Task oriented coping was the only factor that proved minor divergence [9].

CASQ (Coping Across Situations Questionnaire - Seiffge - Krenke, 1995, used by Hanzlova and Macek (2008) facilitates assessing situational impact on coping preferences. It consists of 20 coping strategies and respondents have to mark all strategies they might use in a particular domain. The sample was comprised of adolescents attending schools in Moravia, Czech Republic, ranging from 6th class elementary school to 4th class secondary school. The goal of this study was to identify coping strategies utilised in stressful events. They focused on coping strategies in 8 following domains: school (assessment, relations with schoolmates, quantities of information, etc.), parents (understanding, demands, different views, acceptance, freedom in decision-making, independence, etc.), peers (mutual interests, making friends, accepting an individual in a community, etc.), leisure time (lack of free time, parents' limitation, etc.), opposite gender (meeting a partner, socialising with opposite gender, fear of hurting other person, etc.), self (solitude, appearance, dissimilarities from others, etc.), profession, future. [10]

Ayers a Sandler (1996) used CCSC (Children's Coping Checklist Strategies) questionnaire to determine most commonly resorted school children's coping strategies. It contains 11 coping strategies as follows: Cognitive Decision Making (CDM), Direct Problem Solving (DPS), Seeking Understanding (SU), Positive Cognitive Restructuring (PCR), Expressing Feelings (EF), Physical Release of Emotion (PRE), Distracting Action (DA), Avoidant Action (AA), Cognitive Avoidance (CA), Emotion Focused Support (EFS) and Problem Focused Support (PFS).

The investigated sample contained elementary school participants aged $9-13$. One sample was comprised of younger children 
and the other sample children aged 11 and over. The questionnaire was designed to identify the most frequent coping strategies utilised by children in schools. The investigated sample featured 367 children whose parents are or are being divorced. The main goal of the analysis was to reveal coping styles of children in divorced families. Furthermore, they examined divergence of coping strategies preference in relation to gender and age of a child. The findings suggest that no divergence of coping strategies preference related to gender and age was proved. [1]

\section{Empirical Research of School Stress Levels and its Coping Strategies (CCSC)}

The primary focus of our study was the extent of school stress and its coping styles. CCSC (Children's Coping Checklist Strategies) became an essential tool of our research. The main goal was to analyse a sample of senior elementary school children in order to determine the extent of stress perceived and to identify the most commonly utilised coping strategy. Moreover, the study was aimed to make a comparison of most frequently used coping strategy in respondents with higher stress levels perceived and respondents who perceived lower stress levels.

We presumed the following:

H1: The highest percentage of senior elementary school students perceive considerable extent of stress.

$\mathrm{H} 2$ : The highest percentage of respondents "often or always" utilise "distracting action" (DA) and "avoidant action" (AA).

H3: The highest percentage of girls "often or always" utilise "emotion focused support" (EFS) and the highest percentage of boys "often or always" utilise "physical release of emotion" (PRE).

H4: The selection of coping strategies is influenced by the extent of perceived stress.
H4a: Children with high level of perceived stress will "often or always" utilise "emotion focused support" (EFS) and problem focused support (PFS).

H4b: Children with low level of perceived stress will "often or always" utilise "distracting action" (DA), "avoidant action" (AA) or "cognitive avoidance" (CA).

The investigated sample was composed of 298 children attending Elementary School of St. Gorazd in Puchov (163 girls and 135 boys). The questionnaire was administered in November 2008.

Following methods and methodology were implemented so as to determine the extent of perceived stress and to explore most common coping strategies used by senior elementary school children:

Perceived stress questionnaire [14] - the questionnaire was proposed to determine the extent of stress perceived by school children and adolescents.

The Children's Coping Strategies Checklist (CCSC) - the questionnaire devised by Tim S. Ayers a Irwin N. Sandler (1999) is used to determine most frequently utilised coping strategies.

The Children's Coping Strategies Checklist (CCSC) - the 45item questionnaire contains 11 coping strategies. The question "What did you do when you had a problem last month?" appears after every 10 responses to remind the participants what kind of question they are responding to. The assessment of the questionnaire is based on a 4-point scale.

In order to verify assumption 4 it was inevitable to select respondents with high and low levels of perceived stress. Respondents were divided into two groups according to perceived stress levels; $50 \%$ of children with the highest score were placed in the group of high levels of perceived stress and $50 \%$ of children with the lowest score were placed in the group of low levels of perceived stress.

\begin{tabular}{|l|l|}
\hline Coping strategy & Characteristics \\
\hline Cognitive decision making (CDM) & Planning or thinking about ways to solve the problem and thinking about how it will affect the future. \\
\hline Direct Problem Solving (DPS) & $\begin{array}{l}\text { Efforts to improve the problem situation through changing oneself or others. It does not regard thinking but } \\
\text { executing things. }\end{array}$ \\
\hline Seeking Understanding (SU) & Efforts to find meaning in a problem situation or try to understand it better. \\
\hline Positive cognitive restructuring (PCR) & $\begin{array}{l}\text { Thinking about things in the future with an optimistic manner. Minimizing the problem or the consequences } \\
\text { of the problems. }\end{array}$ \\
\hline Expressing feelings (EF) & Verbal expression of feelings. \\
\hline Physical Release of Emotion (PRE) & Efforts to physically work off feelings with physical exercise, play or efforts to physically relax \\
\hline Distracting action (DA) & $\begin{array}{l}\text { Efforts to avoid thinking about the problem situation by using distracting stimuli, entertainment or some } \\
\text { distracting activity. }\end{array}$ \\
\hline Avoidant action (AA) & Efforts of avoiding the problem by staying away from it or leaving it. \\
\hline Cognitive Avoidance (CA) & Efforts of avoiding the problem by using fantasy. \\
\hline Problem Focused Support (PFS) & The use of other people as resources to assist in seeking solutions to the problem situation. \\
\hline Emotion Focused Support (EFS) & The involvement of other people in listening to feelings or providing understanding to help the person. \\
\hline
\end{tabular}




\section{Analysis and Interpretation}

Assumption $\mathrm{H} 1$ suggests that the investigated sample of children will perceive high stress levels.

Perceived stress levels in students of elementary schools

Table 2

\begin{tabular}{|l|c|}
\hline Stress levels & Number of children (in per cent) \\
\hline High stress levels & $11.8 \%$ \\
\hline Low stress levels & $20.1 \%$ \\
\hline Medium stress levels & $68.1 \%$ \\
\hline
\end{tabular}

Based on the results, we can draw a conclusion that our assumption that high levels of perceived stress will be the most notable in senior elementary school students, was not proved. The findings suggest that senior elementary school students do not perceive stress to such a considerable extent as it was presumed.

It is estimated that approximately $10 \%$ of students perceive high levels of school stress. The estimates are grounded in students' own feelings about school (anxiety, fear) and symptoms induced by stress (stomachache, nausea). [14]

The research was deliberately not conducted before the end of the school term when students perceive higher leves of stress due to exams, tests and assignments deadlines.

The percentage of children perceiving high levels of stress grows considerably in periods when they are exposed to particular pressure, e.g. before an important exam. Hodge et al. (1997) conducted a research focused on a comparison of school stress levels in a sample of 446 students, aged 16-18 in New South Wales, Australia, preparing for graduation exams. Students completed the questionnaire commonly used for identifying risk of mental problems. More than a half of students' results exceeded the risk limit and their values even rose towards the impending exams. [14]

The goal of this study was to explore most commonly used coping strategies of senior elementary school students. Assumption $\mathrm{H} 2$ suggests that the investigated sample of children will mostly deploy "distracting action" (DA) and "avoidant action" (AA) coping strategies.

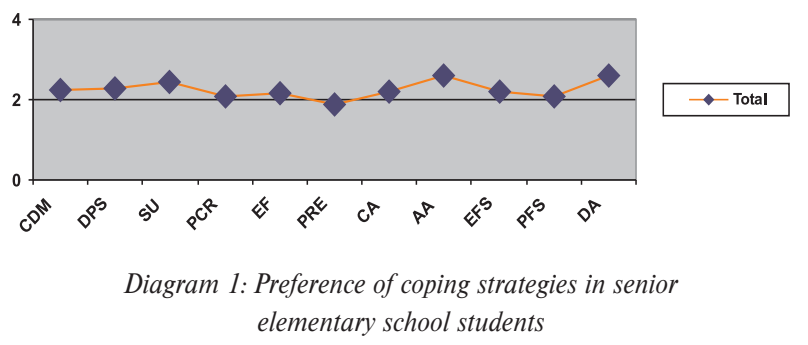

Diagram 1 shows that children tend to avoid problems as most commonly utilised coping strategies are "distracting action" (DA), "avoidant action" (AA). They are used by more than a half of respondents. Another strategy significantly preferred by children is "seeking understanding" (SU). The least favoured strategies were "physical release of emotion" (PRE), "positive cognitive restructuring" (PCR) and "problem focused support" (PFS).

When comparing the results of our research with Amirkhan's results of the research (CSI, Coping Strategy Indicator, 1990), we can conclude that most frequently utilised coping strategy in both cases is "avoidant action" (AA).

On the contrary research carried out by Hanzlova and Macek (2008) by means of CASQ (Coping Situations Questionnaire Seiffge-Krenke, 1995) reveals that the most commonly utilised coping strategy is active problem solving and reflections about possible solutions.

Assumption $\mathrm{H} 3$ suggests that the highest percentage of girls "often or always" utilise "emotion focused support" (EFS) and the highest percentage of boys "often or always" utilise "physical release of emotion" (PRE).

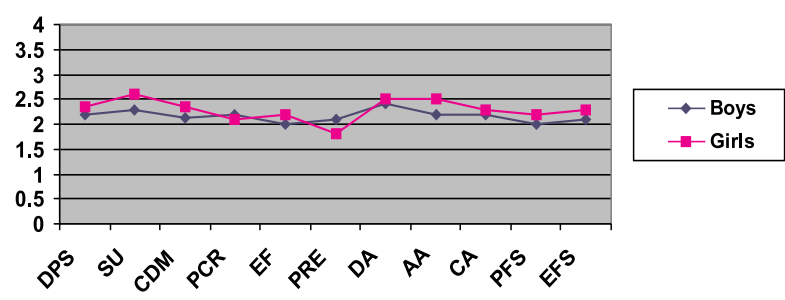

Diagram 2: Preference of coping strategies in relation to gender

Assumption $\mathrm{H} 3$ was not proved as the research revealed that girls "often" utilised "seeking understanding" (SU) and not the presumed coping strategy "emotion focused support" (EFS). Boys "often" use "distracting action" (DA) as a coping strategy instead of presumed "physical release of emotion" (PRE). Both genders favour "seeking understanding" (SU), "distracting action" (DA), "avoidant action" (AA). However, some divergence between boys and girls has been observed in the least utilised coping strategies. Girls declare that the least utilised coping strategies in stressful events are "physical release of emotion" (PRE) and "positive cognitive restructuring" (PCR). Boys' least preferred coping strategies include "expressing feelings" (EF) and "problem focused support” (PFS).

Research carried out by Hanalova and Macek (2008) by means of CASQ (Coping Situations Questionnaire - Seiffge-Krenke, 1995) reveals differences in coping strategies preference in relation to gender.

The outcomes showed that girls tend to reflect on problems and they come up with various solutions to a problem, discuss the 
problem with parents or someone else, seek consolation and support in people with similar problems, they openly speak about the problem and are willing to accept their limitations. It can be summed up that they speak about the problem more frequently, seek social support and respect reality. Boys, on the contrary, tend to conceal their problems and act as if everything was all right, they do not worry about things as they believe that problems will be settled and they tend to resort to alcohol and drugs more often. It can also be noted that girls prefer to seek help to their problem from friends rather than from their parents and boys vice versa.

Assumption $\mathrm{H} 4$ presumes that the selection of coping strategies is influenced by the extent of perceived stress. The basic assumption was subdivided into two partial ones:

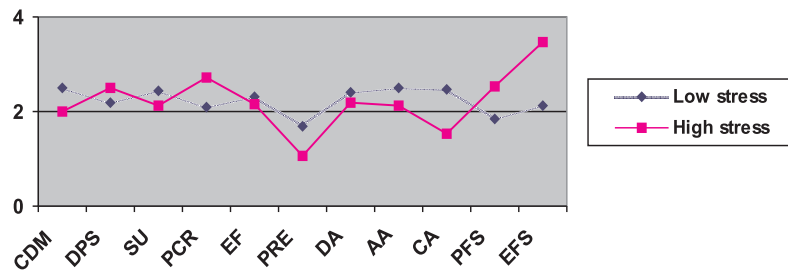

Diagram 3. Selection of coping strategies in relation to the extent of perceived stress

Verification of the the assumption H4a was targeted at finding whether the children who perceive high levels of stress select coping strategies different from those with low levels of stress. We assumed that the children who perceive high levels of stress "often or always" utilise "emotion focused support" (EFS) and "problem focused support" (PFS). Our assumption was based on the hypothesis that the children who perceive high levels of stress will seek help from their parents, friends or acquaintances.

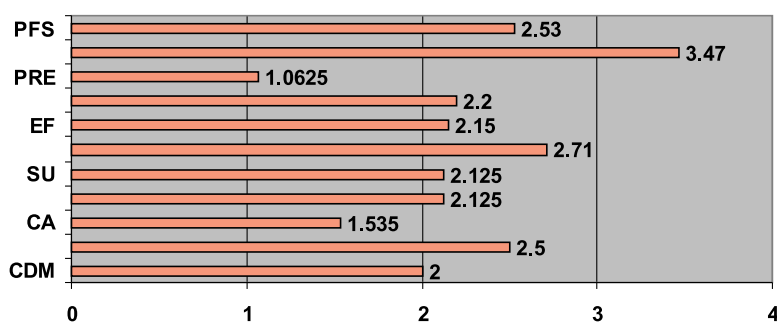

Diagram 4: Coping strategies preference of children who perceive high levels of stress

Based on the analysis of the values of particular items we can draw a conclusion that the most frequently utilised coping strategies are "emotion focused support" (EFS) and "problem focused support" (PFS). The results of our findings thus match our assumption. Children who perceive high levels of stress "almost always" seek help from other people. However, it does not regard direct help; they seek the involvement in listening to feelings or provid- ing understanding to help the person. Another coping strategy rather frequently utilised by children who perceive high levels of stress is "positive cognitive restructuring" (PCR), which means thinking about a problem in a positive manner or minimising the problem. The least utilised coping strategies are "physical release of emotion" (PRE) and "cognitive avoidance" (CA).

Verification of $\mathrm{H} 4 \mathrm{~b}$ assumption was aimed at finding if children perceiving low levels of stress utilise coping strategies different from children with high levels of stress. It was presumed that children perceiving low levels of stress "often or almost always" utilise "avoidant action" (AA), "distracting action" (DA) and "cognitive avoidance" (CA). Our assumption was based on the hypothesis that the children who perceive low levels of stress will not attempt to solve the problem directly but will tend to evade it as they do not assign it great importance.

The findings of our research suggest that the three most frequent coping strategies utilised by children perceiving low levels of stress are evasive strategies. Children perceiving low levels of stress "often" use "distracting action" (DA), "avoidant action" (AA) and "cognitive avoidance" (CA). Based on the results of our research it can be concluded that children who perceive low levels of stress tend to avoid stressful events by choosing to leave, doing something different or escaping reality by means of fantasy. The least utilised coping strategies are "emotion focused support" (EFS) and "problem focused support" (PFS). "Physical release of emotion" (PRE) is another less frequently opted coping strategy. Our assumption that children perceiving low levels of stress will "often" use "distracting action" (DA), "avoidant action" (AA) and "cognitive avoidance" (CA) matches the outcomes of our findings.

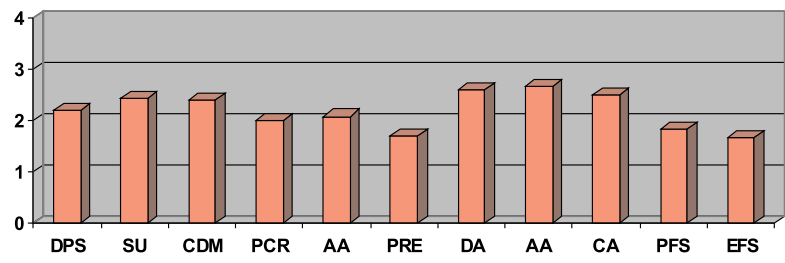

Diagram 5: Coping strategies preference of children who perceive low levels of stress

\section{Conclusion}

Coping with stress and stressful events is of vital importance for pupils. This is, however, in a sharp contrast with the fact that only very little attention is paid to a systematic training of coping with load both in real life and psychological research. The necessity of such a training is only discussed in cases when an individual could not bear the load of a failure at school and resorted to an act of sheer desperation (running away from home, suicidal attempt) [7]

Bratská (Constructive solution and coping with load, 2004) suggests a perspective of a group therapy focused on coping with load 
in order to get acquainted, compare and adopt various techniques in accordance with awareness of possible consequences.

The programme is applicable in miscellaneous groups in nursery schools, elementary schools, secondary schools, universities as wel as at third age universities. The program presents a constructive, efficient and creative solution and coping with load. Moreover, it analyses support of active adaptation to difficult conditions in a social system and development of communication and interpersonal skills. The principal psychological objective of the programme is to enhance social competence of participants with regard to constructive, efficient and creative solution and coping with load. [6]
Changes in reactions to potentially stressful situations at school are desirable outcomes of coping strategies training. The main goal according to Mareš [15, p.563] is to teach pupils wide range of coping strategies, proper assessment of the situation or possibly unteach them generally inappropriate or situationally disadvantaged strategies.

It can be concluded that age-appropriate load with a suitable adopted coping strategy may have a positive impact, it can enhance personality development, it can trigger changes, or it can be a challenge or a chance to achieve something.

\section{References}

[1] AYERS, T. A., SANDLER, I. N.: Manual for the Children's Coping Strategies Checklist \& How I Coped Under Pressure Scale, [on line], Arizona, State prevention Research, [cit. 2008-10-26], http//www.asu.edu/clas/asuprc/, 1999

[2] AYERS, T. A., SANDLER, I. N.: A Dispositional and Situational Assessment of Children's Coping: Testing Alternative models of Coping, J. of Personality, 1996, 64(4), pp. 923-958

[3] BAUMGARTNER, F.: Coping (in Slovak), J. Vyrost \& I. Slamenik (Eds.), Aplikovana socialni psychologie II, Praha, Grada Publishing, 2001, pp. 191-208

[4] BOBACIKOVA, M.: Pupils and School Stress (in Slovak), KPS, Faculty of Science, University of Zilina, Zaverecna bakalarska praca, 2008, p. 57, 2008

[5] BRATSKA, M.: Can we Master Pressure Situations? (in Slovak), Bratislava, Slovenske pedagogicke nakladatelstvo, 1992, p. 147, ISBN 80-08-01592-6

[6] BRATSKA, M.: Intervention Programme Proposing Constructive Solutions and Coping, Pedagogicka revue, 1/2004, vol. 56, pp. 5361, ISSN 1335-1982

[7] CAP, J., MARES, J.: Psychology and the Teacher (in Czech), Praha, Portal, 2001, p. 655, ISBN 80-7178-463-X

[8] FICKOVA, E.: Coping Determinants (in Slovak), Teoreticke pristupy, Ceskoslovanska psychologie, 1/1993, vol. 37, pp. 37-45

[9] FICKOVA, E.: Personality and Coping with Stress (in Slovak), Bratislava, Ustav experimentalnej psychologie SAV, 2001, p. 95, ISBN 80-88910-07-2

[10] HANZLOVA, M., MACEK, P.: Coping Strategies and Styles of Adolescents (in Czech), Psychologia a patopsychologia dietata, 1/2008, pp. 3-22.

[11] KOUBEKOVA, E.: School - Potential Source of Children's Stress (in Slovak), Vychovavatel, 5-3/2000, vol. 44, pp. 10-11

[12] KOUBEKOVA, E.: Everyday Stress in Our Lives (in Slovak), Vychovavatel, 2005, pp. 14-16, ISSN 0139-6919

[13] KRIVOHLAVY, J.: How to Cope with Stress (in Czech), Praha, Grada Avicenum, 1994, p. 190

[14] KYRIACOVA, CH.: Dealing with Educational Problems in School (in Slovak), Praha, Portal, 2003, p. 151, ISBN 80-7178-945-3

[15] MARES, J.: Self-handicapping Coping Strategies(in Czech), Ceskoslovenska psychologie, 4/1999, vol. 45, pp. 311-322

[16] SARMANY-SCHULLER, I.: Reflections on Psychology in Slovakia (in Slovak), Zbornik prispevkov 9. zjazdu slovenskych psychologov, Bratislava, 1999, p. 184, ISBN: 80-85697-50-5. 